CTP TAMU-13/95

hep-th/9503158

\title{
BRST Operators for Higher-spin Algebras 1
}

\author{
H. Lü, C.N. Pope and K.W. Xu \\ Center for Theoretical Physics, Texas A\&M University, College Station, TX 77843-4242
}

\begin{abstract}
In this paper, we construct non-critical BRST operators for matter and Liouville systems whose currents generate two different $W$ algebras. At the classical level, we construct the BRST operators for $W_{2, s}^{\mathrm{M}} \otimes W_{2, s^{\prime}}^{\mathrm{L}}$. The construction is possible for $s=s^{\prime}$ or $s \geq s^{\prime}+2$. We also obtain the BRST operator for $W_{2,4}^{\mathrm{M}} \otimes W_{4}^{\mathrm{L}}$ at the classical level. We use free scalar realisations for the matter currents in the above constructions. At the full quantum level, we obtain the BRST operators for $W_{2, s}^{\mathrm{M}} \otimes W_{2}^{\mathrm{L}}$ with $s=4,5,6$, where $W_{2}$ denotes the Virasoro algebra. For the first and last cases, the BRST operators are expressed in terms of abstract matter and Liouville currents. As a by-product, we obtain the $W_{2,4}$ algebra at $c=-24$ and the $W_{2,6}$ algebra at $c=-2$ and $-\frac{286}{3}$, at which values the algebras were previously believed not to exist.
\end{abstract}

\footnotetext{
${ }^{1}$ Supported in part by the U.S. Department of Energy, under grant DE-FG05-91-ER40633
} 
Owing to the non-linearity of $W$ algebras, BRST methods seem to provide the only reliable way of quantising the associated $W$-string theories. Since the construction of the critical BRST operator for the $W_{3}$ algebra by Thierry-Mieg [1], a number of developments have taken place. Critical BRST operators for more complicated $W$ algebras, including $W_{2, s}$ for $s \leq 8$ [2], $W_{4}$ [4, 5, 6] and $W B_{2}$ [6], have been constructed. In a remarkable development, it was shown in Ref. [7] that a non-critical $W_{3}$ BRST operator can be constructed, involving currents generating two commuting copies of the $W_{3}$ algebra. One copy can be viewed as the matter sector, and the other as the Liouville sector, with the sum of the central charges of the two sectors adding up to the critical value. Subsequently the non-critical $W_{2,4}$ BRST operator was obtained [8]. It is surprising that such constructions can be carried out for systems with non-linear $W$ symmetries, since one cannot simply add the currents of the two copies and obtain a realisation of the algebra. This contrasts with the situation for a system with a linear symmetry, where one merely adds the currents of the matter and Liouville sectors to obtain a new critical realisation of the algebra. For a system with non-linear symmetry, however, the critical and non-critical BRST operators are intrinsically different.

The philosophy behind the construction of the non-critical BRST operators discussed above is that there are two commuting copies of the same $W$ algebra. In this paper, we shall generalise the construction to the case where the currents of the matter sector and Liouville sector generate two different $W$ algebras. The ghost fields of the BRST operator arise from the gauge fixing of the local symmetries generated by the matter currents. Thus the $W$ algebra in the matter sector defines the ghost content of the BRST operator. We shall consider non-critical BRST operators for the $W_{2, s}^{\mathrm{M}} \otimes W_{2, s^{\prime}}^{\mathrm{L}}, W_{2,4}^{\mathrm{M}} \otimes W_{4}^{\mathrm{L}}$ and $W_{2, s}^{\mathrm{M}} \otimes W_{2}^{\mathrm{L}}$ systems, where $W_{2}$ stands for the Virasoro algebra.

To begin, we review some previous results for critical $W_{2, s}$ BRST operators. The $W_{2, s}$ algebra exists at the classical level for all positive integer values of $s$. It is generated by the energy-momentum tensor $T$ and a primary spin-s current $W$. The classical OPE $W(z) W(0)$ is given by

$$
W(z) W(w) \sim \frac{2 T^{s-1}}{(z-w)^{2}}+\frac{\partial T^{s-1}}{z-w} .
$$

The classical BRST operator is given by 3

$$
Q=\oint\left(c(T-s \beta \partial \gamma-(s-1) \partial \beta \gamma-b \partial c)+\gamma W-\partial \gamma \gamma b T^{s-2}\right),
$$

where $(c, b)$ and $(\gamma, \beta)$ are the ghosts and anti-ghosts for the currents $T$ and $W$ respectively. Unlike the case of the $W_{3}$ algebra, this BRST operator cannot, for general values of $s$, be 
extended to a full quantum BRST operator for the abstract $W_{2, s}$ algebra. This is because for general value of $s$, the $W_{2, s}$ algebra exists at the quantum level only for certain discrete values of central charge, which do not include the value needed for criticality. However, it was shown [2, 3] that if one realises the classical currents with free scalars, the resulting classical BRST operator is quantisable. In this realisation, all scalars but one distinguished scalar $\phi$ enter the currents only via their energy-momentum tensor $T_{X}$ [3]:

$$
\begin{aligned}
T & =-\frac{1}{2}(\partial \phi)^{2}+T_{X} \\
W & =\sum_{n=0}^{[s / 2]} s^{-1}(-2)^{-s / 2} 2^{n+1}\left(\begin{array}{c}
s \\
2 n
\end{array}\right)(\partial \phi)^{s-2 n} T_{X}^{n} .
\end{aligned}
$$

In terms of this realisation, the BRST operator can be transformed by a local canonical field redefinition into the following graded form [3]:

$$
\begin{aligned}
Q_{0} & =\oint c(T-s \beta \partial \gamma-(s-1) \partial \beta \gamma-b \partial c), \\
Q_{1} & =\oint \gamma\left((\partial \phi)^{s}+\frac{1}{2} s^{2}(\partial \phi)^{s-2} \beta \partial \gamma\right) .
\end{aligned}
$$

To quantise the classical $W_{2, s}$ string and obtain a critical quantum BRST operator, one can add $\hbar$-dependent quantum corrections to the classical BRST operator. Explicit solutions have been found for all $s \leq 8$ [2, 3], and general existence arguments for one class of solutions for all values of $s$ were given in Refs. [9, 2].

Now we turn to the construction of the non-critical BRST operators for $W_{2, s}^{\mathrm{M}} \otimes W_{2, s^{\prime}}^{\mathrm{L}}$. Again we use the free-scalar realisation (3) for the $W_{2, s}^{\mathrm{M}}$ system, so that the corresponding BRST operator can be written in a graded form, generalising Eqn. (4). By demanding nilpotence at the classical level, we find that the BRST operator is given by $Q=Q_{0}+Q_{1}$ with

$$
\begin{aligned}
Q_{0}= & \oint c\left(T_{\phi}+T_{X}+T_{\mathrm{L}}-s \beta \partial \gamma-(s-1) \partial \beta \gamma-b \partial c\right), \\
Q_{1}= & \oint \gamma\left((\partial \phi)^{s}+\sum_{n=1}^{\left[s^{\prime} / 2\right]} g_{n}\left(s^{\prime}\right)(\partial \phi)^{s-2 n} T_{\mathrm{L}}^{n}+\sum_{n=1}^{\left[s^{\prime} / 2+1\right]} h_{n}\left(s, s^{\prime}\right)(\partial \phi)^{s-2 n} T_{\mathrm{L}}^{n-1} \beta \partial \gamma\right. \\
& \left.+2^{-s^{\prime} / 2} s^{\prime}(\partial \phi)^{s-s^{\prime}} W_{\mathrm{L}}+2^{-1-s^{\prime} / 2} s^{\prime}\left(s-s^{\prime}\right)^{2}(\partial \phi)^{s-s^{\prime}-2} W_{\mathrm{L}} \beta \partial \gamma\right),
\end{aligned}
$$

where the coefficients $g_{n}\left(s^{\prime}\right)$ and $h_{n}\left(s, s^{\prime}\right)$ are given by

$$
\begin{aligned}
g_{n}\left(s^{\prime}\right) & =\frac{(-1)^{n} s^{\prime}\left(s^{\prime}-n-1\right) !}{2^{n} n !\left(s^{\prime}-2 n\right) !} \\
h_{n}\left(s, s^{\prime}\right) & =\frac{(-1)^{n+1} s^{\prime}\left(s^{\prime}-n-1\right) !}{2^{n}(n-1) !\left(s^{\prime}-2 n+2\right) !}\left(s(s-4 n+4)\left(s^{\prime}-n\right)+s^{\prime}\left(s^{\prime}-2\right)(n-1)\right) .
\end{aligned}
$$


The Liouville currents $T_{\mathrm{L}}$ and $W_{\mathrm{L}}$ satisfy the classical $W_{2, s^{\prime}}$ algebra, given by Eqn. (1) with $s$ replaced by $s^{\prime}$. It follows from the last term in the $Q_{1}$ operator (5) that either $s \geq s^{\prime}+2$ or $s=s^{\prime}$. The latter case corresponds to the previously-discussed non-critical $W_{2, s}$ BRST operators. The former case corresponds to the new possibilities that we are considering in this paper. When $s^{\prime}=2$, the $W_{2, s^{\prime}}$ algebra becomes a linear algebra, and the BRST operator (6) is slightly modified: The $W$ current in the Liouville sector enters the $Q_{1}$ operator in the combination $W_{\mathrm{L}}-T_{\mathrm{L}}$, which generates the Virasoro algebra. Thus for the case $s^{\prime}=2$, the BRST operator can be simplified to the one for $W_{2, s}^{\mathrm{M}} \otimes W_{2}^{\mathrm{L}}$, where $W_{2}$ denotes the Virasoro algebra. We find that it is given by

$$
\begin{aligned}
Q_{0} & =\oint c\left(T_{X}+T_{\phi}+T_{\mathrm{L}}-s \beta \partial \gamma-(s-1) \partial \beta \gamma-b \partial c\right) \\
Q_{1} & =\oint \gamma\left((\partial \phi)^{s}+\frac{1}{2} s^{2}(\partial \phi)^{s-2} \beta \partial \gamma-2(\partial \phi)^{s-2} T_{\mathrm{L}}-(s-2)^{2}(\partial \phi)^{s-4} T_{\mathrm{L}} \beta \partial \gamma\right) .
\end{aligned}
$$

Having obtained the classical BRST operators for $W_{2, s}^{\mathrm{M}} \otimes W_{2}^{\mathrm{L}}$ and $W_{2, s}^{\mathrm{M}} \otimes W_{2, s^{\prime}}^{\mathrm{L}}$, we now turn our attention to their quantisation. For generic values of $s$ and $s^{\prime}$, the associated algebras do not close except at discrete sets of values of the central charges. Thus it is not possible to construct abstract quantum BRST operators for generic $s$ and $s^{\prime}$. However, when $s$ and $s^{\prime}$ take their values in the set $\{2,3,4,6\}$ the corresponding algebras close for generic values of central charge. Thus at the quantum level, in addition to the previously constructed critical $W_{2, s}$ BRST operators and non-critical BRST operators with $s=s^{\prime}$, there are four more non-critical BRST operators that can be possibly constructed abstractly, namely, $W_{2,4}^{\mathrm{M}} \otimes W_{2}^{\mathrm{L}}, W_{2,6}^{\mathrm{M}} \otimes W_{2}^{\mathrm{L}}$, $W_{2,6}^{\mathrm{M}} \otimes W_{2,3}^{\mathrm{L}}$ and $W_{2,6}^{\mathrm{M}} \otimes W_{2,4}^{\mathrm{L}}$. In this paper, we construct abstract quantum BRST operators for $W_{2, s}^{\mathrm{M}} \otimes W_{2}^{\mathrm{L}}$ with $s=4,6$. These two BRST operators turn out to be surprisingly simple, with a graded structure $Q=Q_{0}+Q_{1}$ where $Q_{0}^{2}=Q_{1}^{2}=\left\{Q_{0}, Q_{1}\right\}=0$.

For the case $s=4$, we write down the most general possible structure, subject to the requirement that $\{Q, b\}$ gives rise to the standard total matter-plus-ghost energy-momentum tensor, and solve for the coefficients by demanding nilpotence. We find that the BRST operator can be nilpotent provided that the central charges for the matter and Liouville sectors are $c_{\mathrm{M}}=196$ and $c_{\mathrm{L}}=-24$. The BRST operator has two free parameters, which correspond to the freedom to perform canonical transformations on the ghost fields. Choosing the parameters suitably, we find that the BRST operator can be written in a graded form, given by

$$
\begin{aligned}
Q_{0} & =\oint c\left(T_{\mathrm{M}}+T_{\mathrm{L}}-4 \beta \partial \gamma-3 \partial \beta \gamma-b \partial c\right) \\
Q_{1} & =\oint \gamma\left(\sqrt{\frac{130594}{11}} W_{\mathrm{M}}+\frac{113}{132} T_{\mathrm{M}}^{2}+\frac{445}{88} \partial^{2} T_{\mathrm{M}}-\frac{167}{22} T_{\mathrm{M}} \beta \partial \gamma-\frac{1169}{44} \beta \partial^{3} \gamma+\frac{1169}{66} \partial^{2} \beta \partial \gamma\right) .
\end{aligned}
$$


It is interesting to compare this BRST operator with the usual non-critical BRST operator for $W_{2,4}^{\mathrm{M}} \otimes W_{2,4}^{\mathrm{L}}$ at $c_{\mathrm{L}}=-24$. At this particular value of central charge, the $W_{2,4}$ algebra becomes singular. However, we find that one can rescale the spin-4 current such that no coefficients diverge in the OPE $W(z) W(w)$, which is now given by

$$
W(z) W(w) \sim \frac{2 T W-\frac{1}{3} \partial^{2} W}{(z-w)^{2}}+\frac{\partial(T W)-\frac{1}{6} \partial^{3} W}{z-w} .
$$

It should be emphasised that the algebra generated by $T$ and $W$ is consistent and satisfies the Jacobi identity. We find that the usual non-critical BRST operator for $W_{2,4}^{\mathrm{M}} \otimes W_{2,4}^{\mathrm{L}}$ at $c_{\mathrm{L}}=-24$ is given by

$$
Q=Q_{0}+Q_{1}-\oint \gamma\left(\frac{167}{22} W_{\mathrm{L}}+\frac{27889}{484} W_{\mathrm{L}} b \partial \gamma\right)
$$

where the first two terms are precisely given by Eqn. (8). Now it is easy to understand why the BRST operator (8) for $W_{2,4}^{\mathrm{M}} \otimes W_{2}^{\mathrm{L}}$ exists. It follows from the OPE (9) that we can consistently set $W_{\mathrm{L}}$ to zero since it appears in every term on the right hand side of the OPE. Thus the BRST operator (10) will continue to be nilpotent if $W_{\mathrm{L}}$ is set to zero.

There are three other values of the central charge for which the $W_{2,4}$ algebra becomes singular, namely $c=\frac{1}{2},-\frac{68}{7}$ and $-\frac{22}{5}$. For these values, we can also rescale the spin- 4 current such that its OPE with itself has no divergent coefficients. In each case, however, we find that the result fails to satisfy the Jacobi identity. Thus we expect that there is no non-critical BRST operator for $W_{2,4}^{\mathrm{M}} \otimes W_{2,4}^{\mathrm{L}}$ when $c_{\mathrm{L}}$ is equal to any of these three values. By contrast, as we have shown, when $c=-24$ the $W_{2,4}$ algebra is consistent, although degenerate, and we have constructed the associated abstract non-critical BRST operator. The existence of the algebra at $c=-24$ was not seen in the previous results in Refs. [10, 11]. This can be understood as follows. For generic values of central charge, the $W_{2,4}$ algebra can be expressed in the simple form [10, 11]

$$
W \circ W=\frac{c}{4} \mathbf{1}+q W
$$

where

$$
q^{2}=\frac{54(c+24)\left(c^{2}-172 c+196\right)}{(2 c-1)(7 c+68)(5 c+22)} .
$$

In the abbreviated notation of Eqn. (11), the right-hand side denotes the identity operator and the spin-4 current and all their descendants. This expression is however misleading for special values of central charge, since some of the descendant terms have additional central charge dependent factors that can diverge at the special values. In particular, the $W$ descendant 
$T W-\frac{1}{6} \partial^{2} W$ has a coefficient factor $1 /(c+24)$. We can then rescale the $W$ current such that $W \longrightarrow W / \sqrt{c+24}$, and thereby obtain the $W_{2,4}$ algebra at $c=-24$, as given by (9).

The degeneration of the $W_{2,4}$ algebra at $c=-24$ is analogous to the degeneration of the $W_{3}$ algebra at $c=-\frac{22}{5}$. In this latter case, one can again rescale the spin- 3 current, thereby obtaining the OPE

$$
W(z) W(w) \sim \frac{2\left(T^{2}-\frac{3}{10} \partial^{2} T\right)}{(z-w)^{2}}+\frac{\partial T^{2}-\frac{3}{10} \partial^{3} T}{z-w} .
$$

It is easy to verify that $T$ and $W$ define a consistent algebra which satisfies the Jacobi identity. We have verified that it can be used to construct the non-critical BRST operator for $W_{3}^{\mathrm{M}} \otimes W_{3}^{\mathrm{L}}$ with $c_{\mathrm{L}}=-\frac{22}{5}$. However, in this case one cannot obtain a non-critical BRST operator for $W_{3}^{\mathrm{M}} \otimes W_{2}^{\mathrm{L}}$ by setting the current $W_{\mathrm{L}}$ to zero, since the right hand side of Eqn. (13) does not vanish. In fact we have verified that no $W_{3}^{\mathrm{M}} \otimes W_{2}^{\mathrm{L}}$ BRST operator exists.

For the case of $s=6$, we make the assumption that the BRST operator has the same graded structure, and we find that it is nilpotent when $c_{\mathrm{M}}=390, c_{\mathrm{L}}=-2$ or $c_{\mathrm{M}}=\frac{1450}{3}, c_{\mathrm{L}}=-\frac{286}{3}$. For the first case, the BRST operator is given by

$$
\begin{aligned}
Q_{0}= & \oint c\left(T_{\mathrm{M}}+T_{\mathrm{L}}-6 \beta \partial \gamma-5 \partial \beta \gamma-b \partial c\right), \\
Q_{1}= & \oint \gamma\left(2448 \sqrt{\frac{41149461318}{13}} W_{\mathrm{M}}+4282 T_{\mathrm{M}}^{3}+\frac{1390837}{13} \partial^{2} T_{\mathrm{M}} T_{\mathrm{M}}+\frac{1038100}{13} \partial T_{\mathrm{M}} \partial T_{\mathrm{M}}\right. \\
& +\frac{6815257}{39} \partial^{4} T_{\mathrm{M}}-\frac{1032462}{13} T_{\mathrm{M}}^{2} \beta \partial \gamma+\frac{4301925}{13} \partial^{2} T_{\mathrm{M}} \beta \partial \gamma \\
& +\frac{16634110}{13} \partial T_{\mathrm{M}} \partial \beta \partial \gamma+\frac{6653644}{13} T_{\mathrm{M}} \partial^{2} \beta \partial \gamma-\frac{9980466}{13} T_{\mathrm{M}} \beta \partial^{3} \gamma-1433975 \partial^{4} \beta \partial \gamma \\
& \left.+2581155 \partial^{2} \beta \partial^{3} \gamma-1433975 \beta \partial^{5} \gamma-1720770 \partial \beta \beta \partial^{2} \gamma \partial \gamma\right) .
\end{aligned}
$$

For the latter case, $Q_{0}$ takes an identical form to $Q_{0}$ in Eqn. (14), and the $Q_{1}$ operator is given by

$$
\begin{aligned}
Q_{1}= & \oint \gamma\left(136 \sqrt{\frac{4609647394209}{483799}} W_{\mathrm{M}}-\frac{3557034}{483799} T_{\mathrm{M}}^{3}-\frac{89012250}{483799}\left(\partial T_{\mathrm{M}}\right)^{2}-\frac{98014833}{483799} \partial^{2} T_{\mathrm{M}} T_{\mathrm{M}}\right. \\
& -\frac{103809276}{483799} \partial^{4} T_{\mathrm{M}}+1746 T_{\mathrm{M}} \partial \beta \partial^{3} \gamma+162\left(T_{\mathrm{M}}\right)^{2} \beta \partial \gamma-1224 T_{\mathrm{M}} \partial^{2} \beta \partial \gamma \\
& \left.-1215 \partial^{2} T_{\mathrm{M}} \beta \partial \gamma+2645 \beta \partial^{5} \gamma+6675 \partial^{2} \beta \partial^{3} \gamma+4240 \partial^{4} \beta \partial \gamma-1350 \partial \beta \beta \partial^{2} \gamma \partial \gamma\right) .
\end{aligned}
$$

The existence of the BRST operators (14) and (15) can be understood from the fact that the $W_{2,6}$ algebra becomes degenerate when $c=-2$ and $c=-\frac{286}{3}$, analogous to the case of $W_{2,4}$ at $c=-24$. At $c=-2$, we find that the OPE of the spin-6 current with itself gives rise only to a null current:

$$
W(z) W(w) \sim \frac{2 \Lambda}{(z-w)^{2}}+\frac{\partial \Lambda}{z-w}
$$


where $\Lambda=T^{2} W-\frac{5}{9} T \partial^{2} W+\frac{9}{19} \partial T \partial W-\frac{17}{6} \partial^{2} T W+\frac{1}{36} \partial^{4} W$. At $c=-\frac{286}{3}$, we find that the OPE of the spin-6 current $W$ with itself contains only $W$ and its descendants:

$$
\begin{aligned}
& W(z) W(w) \sim \\
& \frac{W}{(z-w)^{6}}+\frac{1}{2} \frac{\partial W}{(z-w)^{5}}+\frac{7}{52} \frac{\partial^{2} W}{(z-w)^{4}}+\frac{1}{39} \frac{\partial^{3} W}{(z-w)^{3}}+\frac{1}{260} \frac{\partial^{4} W}{(z-w)^{2}}+\frac{1}{2080} \frac{\partial^{5} W}{z-w} \\
& -\frac{9}{35} \frac{\Lambda_{1}}{(z-w)^{4}}-\frac{9}{70} \frac{\partial \Lambda_{1}}{(z-w)^{3}}-\frac{81}{2380} \frac{\partial^{2} \Lambda_{1}}{(z-w)^{2}}-\frac{3}{476} \frac{\partial^{3} \Lambda_{1}}{z-w}+\frac{27}{700} \frac{\Lambda_{2}}{(z-w)^{2}}+\frac{27}{1400} \frac{\partial \Lambda_{2}}{z-w},
\end{aligned}
$$

where $\Lambda_{1}=T W-\frac{3}{26} \partial^{2} W$ and $\Lambda_{2}=T^{2} W-\frac{35}{153} T \partial^{2} W-\frac{2}{153} \partial T \partial W-\frac{29}{102} \partial^{2} T W+\frac{7}{612} \partial^{4} W$. Thus in the BRST operator for $W_{2,6}^{\mathrm{M}} \otimes W_{2, s}^{\mathrm{L}}$, we can consistently set the spin-6 Liouville current $W_{\mathrm{L}}$ to zero when $c_{\mathrm{L}}=-2$ and when $c_{\mathrm{L}}=-\frac{286}{3}$, giving rise to the BRST operators (14) and (15) respectively.

Having obtained the non-critical abstract BRST operators for $W_{2, s}^{\mathrm{M}} \otimes W_{2}^{\mathrm{L}}$ with $s=4$ and 6 , it is interesting to investigate the implications for the corresponding string theories. We can realise the matter $W_{2, s}^{\mathrm{M}}$ currents in terms of free scalars, with the classical terms given by Eqn. (3). For $s=4$ and both the above cases for $s=6$, it turns out that the central charge required by criticality implies that the central charge of the effective energy-momentum tensor $T_{X}$ is $c_{X}=26$. Note that the Liouville current appears only in $Q_{0}$ in the abstract BRST operators (8) and (14), whilst in the BRST operator in terms of scalar realisations, the Liouville current appears both in the $Q_{0}$ and $Q_{1}$ operators. We have verified for $s=4$ that in terms of the free-scalar realisation, the BRST operator (7) with its quantum corrections can be converted into the BRST operator (8) by a local canonical field redefinition.

Now let us turn our attention to the quantisation of the non-critical BRST operators for $W_{2, s}^{\mathrm{M}} \otimes W_{2}^{\mathrm{L}}$ with $s$ taking values other than 4 and 6 . In these cases, we have to start with a specific realisation for the classical $W_{2, s}$ algebra, since at the quantum level it fails to satisfy the Jacobi identify for generic values of central charge except when $s=4,6$. We find that for these non-critical BRST operators, just as in the case of the critical $W_{2, s}$ BRST operators discussed previously [2, 3], nilpotence can be achieved at the quantum level even when the corresponding quantum $W_{2, s}$ algebra does not exist. The classical BRST operators for $W_{2, s}^{\mathrm{M}} \otimes W_{2}^{\mathrm{L}}$ in terms of scalar realisations are given by Eqn. (7). To quantise the BRST operators, we can add all possible $\hbar$-dependent quantum corrections and demand nilpotence. We have studied the case of $s=5$ in detail. Assuming the graded form of the BRST operator is preserved at the quantum level, we find by explicit computation that there is one solution. It is given by

$$
Q_{0}=\oint c\left(T_{X}-\frac{1}{2}(\partial \phi)^{2}+T_{\mathrm{L}}-6 \beta \partial \gamma-5 \partial \beta \gamma-b \partial c\right)
$$




$$
\begin{aligned}
Q_{1}= & \oint \gamma\left((\partial \phi)^{5}-2(\partial \phi)^{3} T_{\mathrm{L}}+\frac{25}{2}(\partial \phi)^{3} \beta \partial \gamma-9 \partial \phi T_{\mathrm{L}} \beta \partial \gamma-\frac{65}{2 \sqrt{2}} \partial^{2} \phi(\partial \phi)^{3}\right. \\
& +\frac{165}{4}\left(\partial^{2} \phi\right)^{2} \partial \phi+\frac{175}{8} \partial^{3} \phi(\partial \phi)^{2}-\frac{265}{8 \sqrt{2}} \partial^{3} \phi \partial^{2} \phi-\frac{55}{4 \sqrt{2}} \partial^{4} \phi \partial \phi+\frac{53}{32} \partial^{5} \phi \\
& +\frac{9}{\sqrt{2}}(\partial \phi)^{2} \partial T_{\mathrm{L}}+\frac{21}{\sqrt{2}} \partial^{2} \phi \partial \phi T_{\mathrm{L}}-\frac{9}{4} \partial \phi \partial^{2} T_{\mathrm{L}}-\frac{27}{4} \partial^{2} \phi \partial T_{\mathrm{L}}-\frac{19}{4} \partial^{3} \phi T_{\mathrm{L}} \\
& +\frac{\sqrt{2}}{16} \partial^{3} T_{\mathrm{L}}-\frac{45}{2 \sqrt{2}}(\partial \phi)^{2} \partial \beta \partial \gamma+\frac{75}{2 \sqrt{2}}(\partial \phi)^{2} \beta \partial^{2} \gamma-\frac{45}{2} \partial \phi \partial \beta \partial^{2} \gamma-\frac{25}{4} \partial^{2} \phi \beta \partial^{2} \gamma \\
& \left.-\frac{65}{8} \partial^{2} \phi \partial \beta \partial \gamma-\frac{21}{2 \sqrt{2}} T_{\mathrm{L}} \beta \partial^{2} \gamma-\frac{15}{2 \sqrt{2}} \partial T_{\mathrm{L}} \beta \partial \gamma-\frac{65}{8 \sqrt{2}} \beta \partial^{4} \gamma-\frac{65}{4 \sqrt{2}} \partial \beta \partial^{3} \gamma\right) .
\end{aligned}
$$

The central charge for the effective energy-momentum tensor is again $c_{X}=26$. We expect that the classical BRST operators (7) for $W_{2, s}^{\mathrm{M}} \otimes W_{2}^{\mathrm{L}}$ are quantisable for all values of $s$, leading in each case to a string with an effective 26 dimensional spacetime.

Next, we consider the non-critical BRST operator for the $W_{2,4}^{\mathrm{M}} \otimes W_{4}^{\mathrm{L}}$ system. Owing to the complexity of the $W_{4}$ algebra, we have only considered this BRST operator at the classical level. Classically, the $W_{4}$ currents in the Liouville sector, namely $T_{\mathrm{L}}, W_{\mathrm{L}}$ and $U_{\mathrm{L}}$ with spins 2 , 3 and 4 respectively, have the following OPEs amongst the higher-spin currents:

$$
\begin{aligned}
W_{\mathrm{L}}(z) W_{\mathrm{L}}(w) & \sim \frac{\frac{1}{3} T_{\mathrm{L}}^{2}+2 U_{\mathrm{L}}}{(z-w)^{2}}+\frac{\frac{1}{6} \partial\left(T_{\mathrm{L}}\right)^{2}+\partial U_{\mathrm{L}}}{z-w}, \\
W_{\mathrm{L}}(z) U_{\mathrm{L}}(w) & \sim \frac{\frac{5}{3} T_{\mathrm{L}} W_{\mathrm{L}}}{(z-w)^{2}}+\frac{\frac{2}{3} \partial\left(T_{\mathrm{L}} W_{\mathrm{L}}\right)}{z-w} \\
U_{\mathrm{L}}(z) U_{\mathrm{L}}(w) & \sim \frac{\frac{2}{9} T_{\mathrm{L}}^{3}+2\left(W_{\mathrm{L}}\right)^{2}}{(z-w)^{2}}+\frac{\frac{1}{9} \partial\left(T_{\mathrm{L}}\right)^{3}+\partial\left(W_{\mathrm{L}}\right)^{2}}{z-w} .
\end{aligned}
$$

In terms of a free-scalar realisation of the $W_{2,4}$ algebra of the form (3), we find that the classical $W_{2,4}^{\mathrm{M}} \otimes W_{4}^{\mathrm{L}}$ non-critical BRST operator takes the graded form

$$
\begin{aligned}
Q_{0}= & \oint c\left(T_{X}+T_{\phi}+T_{\mathrm{L}}-4 \beta \partial \gamma-3 \partial \beta \gamma-b \partial c\right) \\
Q_{1}= & \oint \gamma\left((\partial \phi)^{4}+8(\partial \phi)^{2} \beta \partial \gamma-\frac{4}{3}(\partial \phi)^{2} T_{\mathrm{L}}-\frac{4}{3} U_{\mathrm{L}}\right. \\
& \left.+\frac{2}{9}\left(T_{\mathrm{L}}\right)^{2}-\frac{4 \sqrt{2}}{3} \partial \phi W_{\mathrm{L}}-\frac{16}{9} T_{\mathrm{L}} \beta \partial \gamma\right) .
\end{aligned}
$$

To summarise, we have studied in this paper the construction of the non-critical BRST operators whose matter and Liouville currents generate two different $W$ algebras. At the classical level, we obtained such BRST operators for $W_{2, s}^{\mathrm{M}} \otimes W_{2, s^{\prime}}^{\mathrm{L}}$. The construction is possible for $s=s^{\prime}$ or $s \geq s^{\prime}+2$. We also obtained the classical BRST operator for $W_{2,4}^{\mathrm{M}} \otimes W_{4}^{\mathrm{L}}$. We used free-scalar realisations for the matter currents, and the corresponding BRST operators have a graded form. It would be interesting to extend these results to the quantum level. However, in general the computations become unmanageably complicated. We have explicitly constructed the BRST operators for $W_{2, s}^{\mathrm{M}} \otimes W_{2}^{\mathrm{L}}$ with $s=4,5,6$ at the quantum level. For the first and the 
third cases, we obtained the BRST operators in terms of abstract matter and Liouville currents. This is not possible when $s=5$ since the $W_{2,5}$ algebra does not exist at the quantum level for the value of central charge required by the criticality. We started with the classical BRST

operator for $W_{2,5}^{\mathrm{M}} \otimes W_{2}^{\mathrm{L}}$ with the matter currents realised in terms of free scalars, and obtained the nilpotent quantum BRST operator by adding $\hbar$-dependent corrections. This construction should presumably work for all values of $s$. In terms of the free-scalar realisations for the matter currents, the central charge for the effective energy-momentum tensor $T_{X}$ is $c_{X}=26$ for all the cases $s=4,5,6$. We expect that it will be true also for higher values of $s$.

It is of interest to extend our results to more general classes of $W$ algebras. The classification of possible BRST operators that can be built becomes equally as interesting as the classification of the $W$ algebras themselves. For any algebra $W$, we expect that there should exist a critical BRST operator and a non-critical BRST operator for $W^{\mathrm{M}} \otimes W^{\mathrm{L}}$ at the classical level. If the algebra exists also at the quantum level for generic values of central charge, we expect that we can obtain the quantum BRST operator abstractly; otherwise, the quantisation has to be carried out in terms of a specific realisation. If one allows two different $W$ algebras for the matter and Liouville sectors, the possibilities become more numerous. Although we have constructed the classical BRST operators for a large class of $W$ algebras, the quantisability of these BRST operator remains to be understood. We obtained the full quantum BRST operators for $W_{2, s}^{\mathrm{M}} \otimes W_{2}^{\mathrm{L}}$ with $s=4,5,6$. For the cases $s=4$ and 6 , the existence of the quantum BRST operators can be understood as special cases of $W_{2, s}^{\mathrm{M}} \otimes W_{2, s}^{\mathrm{L}}$ where the spin- 4 or spin- 6 Liouville currents can be consistently set to zero at the special values of central charge that arose in our construction. For the $s=5$ case, on the other hand, there is no such explanation. In view of the complexity of the computations, the understanding of the general structure of the BRST operators requires a less empirical approach.

\section{Acknowledgements}

We are grateful to R. Blumenhagen and H. Kausch for discussions.

\section{References}

[1] J. Thierry-Mieg, Phys. Lett. B197 (1987) 368. 
[2] H. Lü, C.N. Pope and X.J. Wang, Int. J. Mod. Phys. A9 (1994) 1527; H. Lü, C.N. Pope, X.J. Wang and S.C. Zhao, Phys. Lett. B327 (1994) 241.

[3] H. Lü, C.N. Pope, K. Thielemans, X.J. Wang and K.W. Xu, Quantising higher-spin string theories, preprint, hep-th/9410005, to appear in Int. J. Mod. Phys.

[4] K. Hornfeck, Phys. Lett. B315 (1993) 287.

[5] E. Bergshoeff, H.J. Boonstra, S. Panda and M. de Roo, Nucl. Phys. B411 (1994) 717.

[6] C.J. Zhu, Nucl. Phys. B418 (1994) 379.

[7] M. Berschadsky, W. Lerche, D. Nemeschansky and N.P. Warner, Phys. Lett. B292 (1992) 35; Nucl. Phys. B401 (1993) 304.

[8] H. Lü, C.N. Pope, X.J. Wang and S.C. Zhao, Class. Quant. Grav. 11 (1994) 939.

[9] M.D. Freeman and P.C. West, Phys. Lett. B324 (1994) 322.

[10] H.G. Kausch and G.M.T. Watts, Nucl. Phys. B354 (1991) 740.

[11] R. Blumenhagen, M. Flohr, A. Kliem, W. Nahm, A. Rechnagel and R. Varnhagen, Nucl. Phys. B361 (1991) 255. 